University of Nebraska - Lincoln

DigitalCommons@University of Nebraska - Lincoln

Faculty Publications, Department of Physics and Astronomy

Research Papers in Physics and Astronomy

4-3-2006

\title{
Temperature dependence of magnetoresistance in magnetic tunnel junctions with different free layer structures
}

L. Yuan

University of Nebraska-Lincoln

Sy_Hwang Liou

University of Nebraska-Lincoln, sliou@unl.edu

Dexin Wang

NVE Corporation, Eden Prairie, Minnesota

Follow this and additional works at: https://digitalcommons.unl.edu/physicsfacpub

Part of the Physics Commons

Yuan, L.; Liou, Sy_Hwang; and Wang, Dexin, "Temperature dependence of magnetoresistance in magnetic tunnel junctions with different free layer structures" (2006). Faculty Publications, Department of Physics and Astronomy. 30.

https://digitalcommons.unl.edu/physicsfacpub/30

This Article is brought to you for free and open access by the Research Papers in Physics and Astronomy at DigitalCommons@University of Nebraska - Lincoln. It has been accepted for inclusion in Faculty Publications, Department of Physics and Astronomy by an authorized administrator of DigitalCommons@University of Nebraska Lincoln. 


\title{
Temperature dependence of magnetoresistance in magnetic tunnel junctions with different free layer structures
}

\author{
L. Yuan and S. H. Liou \\ Department of Physics and Astronomy and Center for Materials Research and Analysis, University of Nebraska-Lincoln, \\ Lincoln, Nebraska 68588-0111, USA \\ Dexin Wang \\ NVE Corporation, 11409 Valley View Road, Eden Prairie, Minnesota 55344, USA
}

(Received 10 November 2005; revised manuscript received 13 January 2006; published 3 April 2006)

\begin{abstract}
The temperature and bias voltage dependence of magnetoresistance and the resistance of two types of magnetic tunnel junction (MTJ) samples were studied. These two types of MTJ samples have different free layer structures, while having the same pinned layer structures and with the same material for free and reference layers. The layer structure for type 1 MTJs is $80 \mathrm{Ru}-8 \mathrm{CoFeB}-15 \mathrm{Al}_{2} \mathrm{O}_{3}-50 \mathrm{CoFeB}-9 \mathrm{Ru}-54 \mathrm{FeCo}-$ $350 \mathrm{CrMnPt}$ (in angstroms). The layer structure for type $2 \mathrm{MTJ}$ is $80 \mathrm{Ru}-40 \mathrm{CoFeB}-50 \mathrm{RuTa}-40 \mathrm{CoFeB}-$ $15 \mathrm{Al}_{2} \mathrm{O}_{3}-50 \mathrm{CoFeB}-9 \mathrm{Ru}-54 \mathrm{FeCo}-350 \mathrm{CrMnPt}$. The tunneling magnetoresistance (TMR) ratio $\left[\left(R_{A P}-R_{P}\right) / R_{P}\right]$ is about $26 \%$ and $69 \%$ at room temperature for type 1 and type 2 MTJs, respectively. A TMR as high as $107 \%$ has been observed for type 2 MTJ samples at $13 \mathrm{~K}$. By analysis of the voltage and temperature dependence of the resistance and magnetoresistance in these MTJs, we discuss the effects of the magnetic behavior of the free layers, barrier qualities, and barrier interfaces. The results clearly indicate that the micromagnetization orientation at the interface between the free layer and the barrier layer is one of the important factors that determines the TMR ratio.
\end{abstract}

DOI: 10.1103/PhysRevB.73.134403

PACS number(s): 75.70.-i, 85.70.Ay, 85.70.Kh, 72.25.-b

\section{INTRODUCTION}

Magnetic tunnel junctions (MTJs) have been investigated extensively because of their fundamental interest and potential applications to magnetic random access memory (MRAM), read heads, and other magnetic devices. The basic structure of the MTJ is a sandwich of two ferromagnetic layers separated by a thin insulating layer. With an applied magnetic field, the electrical resistance changes with the relative magnetization orientation of the two ferromagnetic layers. ${ }^{1-3}$ The tunneling magnetoresistance (TMR) ratio is defined as $\left(R_{A P}-R_{P}\right) / R_{P}$, where $R_{A P}$ and $R_{P}$ are the resistances for antiparallel $(A P)$ and parallel $(P)$ alignment of the magnetic moments, respectively.

Julliere's model assumes that the TMR is determined by the spin polarization (SP) of the total electronic density of states (DOS) of the ferromagnetic layers near the Fermi surface, and defines the TMR as TMR $=2 P_{1} P_{2} /\left(1-P_{1} P_{2}\right)$, where $P_{1}$ and $P_{2}$ are the spin polarizations associated with the two electrodes as measured using the Tedrow-Meservey technique. ${ }^{4}$ Half-metallic ferromagnetism is studied because of its $100 \%$ spin polarization..$^{5-9}$ The MTJ with manganese perovskites ferromagnetic layer has been observed to have more than $400 \%$ TMR at low temperature utilizing $\mathrm{SrTiO}_{3}$, $\mathrm{PrBaCu}_{2.8} \mathrm{Ga}_{0.2} \mathrm{O}_{7}$, or $\mathrm{CeO}_{2}$ barriers. ${ }^{10,11}$ However, the TMR ratio in these MTJs is still small at room temperature due to the complicated surface and interface of these materials. Currently, conventional MTJs are still based on $3 d$ ferromagnetic metals that show a high TMR ratio well above room temperature.

The TMR value is also dependent on the insulating barrier layer. Improvement in the barrier quality and metal/barrier interfaces results in the enhancement of the TMR value. ${ }^{12,13}$ With an improved barrier fabrication process, the pinholefree and fewer impurities barrier causes the TMR to reach $\sim 70 \%$ at room temperature for an amorphous $\mathrm{Al}_{2} \mathrm{O}_{3}$ barrier. ${ }^{14}$ Recently, a TMR above $200 \%$ has been detected at room temperature by changing amorphous $\mathrm{Al}_{2} \mathrm{O}_{3}$ barrier to crystalline $\mathrm{MgO}$ tunnel barrier. ${ }^{15,16}$

In this paper, we focus on the effect of the magnetic microstructure in the free layer on the TMR ratio. We studied two types of MTJ samples with a different free layer structure while keeping the other conditions as similar as possible (i.e., same free layer material, same pinned layer material and structures, and same barrier oxidation process). The schematic diagrams of these two MTJs are shown in Fig. 1. Because the material for the free layer is the same for both types, the SP should be the same for these two types of

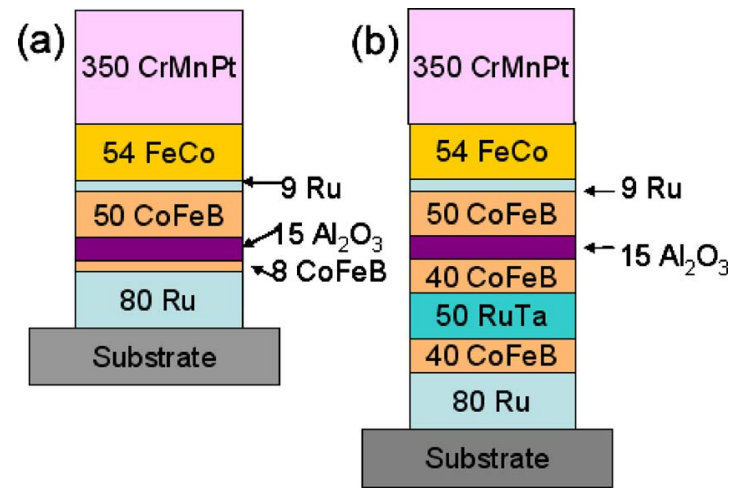

FIG. 1. (Color online) The layer structures of (a) type 1 and (b) type 2 MTJ samples. 
samples. Also, the barrier preparation process is the same; therefore, the barrier quality should be quite similar. However, very different experimental TMR ratios are found for these two types of samples, $26 \%$ and $69 \%$, respectively. By analysis of the voltage dependence of conductance and the temperature dependence of resistance and magnetoresistance in these MTJs, we discuss the detailed effect of magnetic microstructures in the free layers and/or at the interface between the free layer and the barrier layer on TMR behaviors. We show that for different free layer structures, even the same material may have quite different magnetization behaviors, which result in quite different TMR ratios.

\section{EXPERIMENT}

Spin-dependent tunneling (SDT) wafers were deposited using dc magnetron sputtering in a Sharmrock system with a base pressure lower than $1.0 \times 10^{-7}$ Torr. Two types of SDT wafers are shown in Fig. 1. The layer structure of type 1 MTJ samples is $80 \mathrm{Ru}-8 \mathrm{CoFeB}-15 \mathrm{Al}_{2} \mathrm{O}_{3}-50 \mathrm{CoFeB}-9 \mathrm{Ru}-54 \mathrm{FeCo}-$ $350 \mathrm{CrMnPt}$ (in angstroms) [Fig. 1(a)]. The layer structure of type 2 MTJ samples is $80 \mathrm{Ru}-40 \mathrm{CoFeB}-50 \mathrm{RuTa}-40 \mathrm{CoFeB}-15$ $\mathrm{Al}_{2} \mathrm{O}_{3}-50 \mathrm{CoFeB}-9 \mathrm{Ru}-54 \mathrm{FeCo}-350 \mathrm{CrMnPt}$ [Fig. 1(b)]. The antiferromagnetic $\mathrm{CrMnPt}$ layer for pinning is at the top. A $\mathrm{Co}_{60} \mathrm{Fe}_{20} \mathrm{~B}_{20}$ (at \%) target and a $\mathrm{Co}_{40} \mathrm{Fe}_{60}$ target were used for the magnetic layer depositions. The $\mathrm{Al}_{2} \mathrm{O}_{3}$ barrier was formed by depositing a layer of about $12 \AA$ thick metallic $\mathrm{Al}$ and then oxidizing it in a plasma of $\mathrm{Ar} / \mathrm{O}_{2}$. From the transmission electron microscopy (TEM) pictures, the thickness of the $\mathrm{Al}_{2} \mathrm{O}_{3}$ is estimated to be about $15 \AA .{ }^{14} \mathrm{~A}$ magnetic field of 50 Oe was applied during the magnetic layer deposition to induce the easy axis. Annealing was done in forming gas at a temperature of $250{ }^{\circ} \mathrm{C}$ for $1 \mathrm{~h}$ with an applied field of 4 kOe to align the pinning structure. ${ }^{17}$ The junctions were fabricated using photolithographic techniques to pattern the pinned and free layers separately, with one layer of metal to connect the junctions and test pads. ${ }^{18}$ The difference between these two types of MTJ samples is only their free layer structure. For each type, there are many junctions with different junction areas. The resistance area product (RAP) of type 1 MTJs is about $120 \mathrm{M} \Omega \mu \mathrm{m}^{2}$, and for type 2 is about $40 \mathrm{M} \Omega \mu \mathrm{m}^{2}$. The behaviors of MTJs with the same type of structures are similar and independent of their shape. We choose the results of one sample for each type.

\section{RESULTS}

\section{A. Temperature dependence of magnetoresistance}

The magnetotransport properties of the MTJs have been measured with a constant $10 \mathrm{mV}$ bias voltage at various temperatures between 13 and $300 \mathrm{~K}$. For the MTJs, the minor loops are more interesting for practical applications, where only the magnetization of the free layer is switched while the pinned layer remains nearly unaffected. The minor loops were obtained by sweeping an external field of 200 Oe for type 1 and 100 Oe for type 2 devices in the direction along the easy axis of the magnetizations. Figure 2 shows the MR minor loops for type 1 and type 2 MTJ samples at different temperatures. The RAP values at parallel $(P)$ and antiparallel
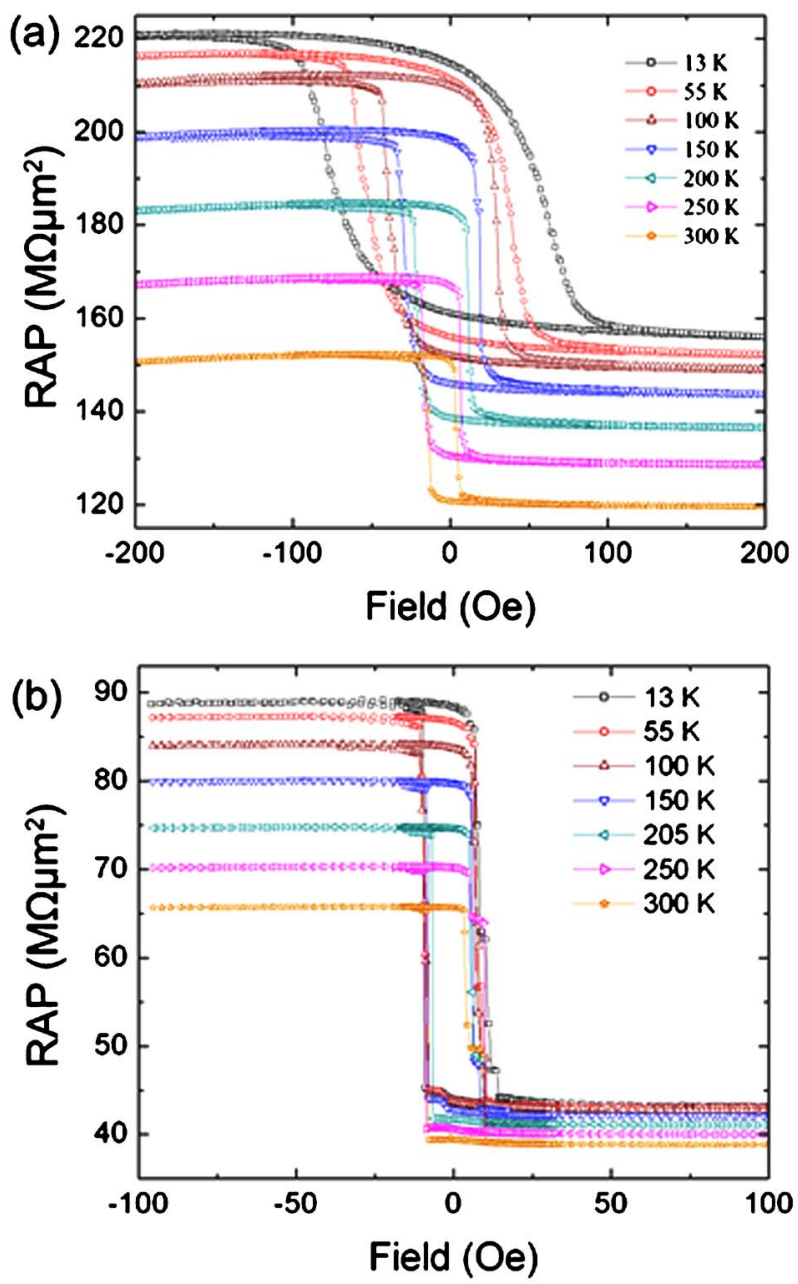

FIG. 2. (Color online) Magnetoresistance transverse loops for (a) type 1 and (b) type 2 MTJs between temperature 13 and $300 \mathrm{~K}$.

$(A P)$ states both increase when the temperature decreases for both types of samples. At room temperature, the type 1 MTJ's RAP in the $P$ state $\left(R_{P}\right)$ is $120.1 \mathrm{M} \Omega \mu \mathrm{m}^{2}$ and the RAP in $A P$ state $\left(R_{A P}\right)$ is $152.8 \mathrm{M} \Omega \mu \mathrm{m}^{2}$; at $13 \mathrm{~K}, R_{P}$ and $R_{A P}$ increase to 155.9 and $220.7 \mathrm{M} \Omega \mu \mathrm{m}^{2}$, respectively. For type 2 MTJ, $R_{P}$ and $R_{A P}$ are 38.7 and $65.3 \mathrm{M} \Omega \mu \mathrm{m}^{2}$ at $300 \mathrm{~K}$ and increase to 43.2 and $88.7 \mathrm{M} \Omega \mu \mathrm{m}^{2}$ at $13 \mathrm{~K}$, respectively.

For both type 1 and type 2, at room temperature the resistance transition between $P$ and $A P$ states happens at about 9 Oe and the transition is very sharp, but their transition behavior is quite different at lower temperatures. We define the squareness $(S)$ of the MR loop as $S=\left(R_{0}-R_{m}\right) /\left(R_{A P}\right.$ $\left.-R_{m}\right)$, where $R_{m}=\left(R_{A P}+R_{P}\right) / 2$ and $R_{0}$ is the resistance at zero fields after saturation in the AP state. From Fig. 3, we observe that the $S$ values of type 1 MTJs did not have a significant change until the temperature decreased to under around $70 \mathrm{~K}$ and that the $S$ values of type 2 samples stay about the same. The low squareness of type 1 samples at lower temperature indicates that the magnetization of the free layer of type 1 is not switched with the field as homogeneously as that of the type 2 .

The coercivity fields $\left(H_{c}\right)$ of both types of samples increase as temperature decreases (Fig. 3). The $H_{c}$ change with 


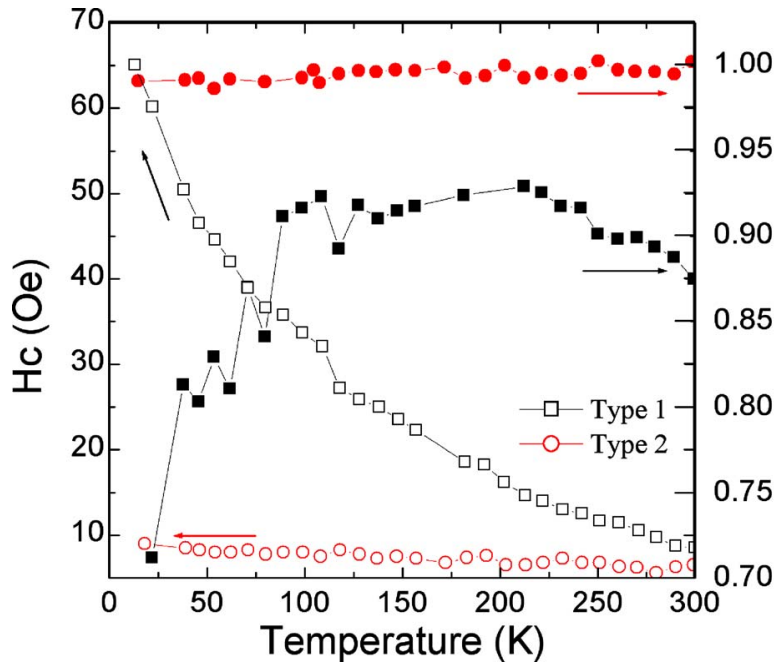

FIG. 3. (Color online) The squareness $(S)$ and hysteresis field $\left(H_{c}\right)$ of type 1 and type 2 MTJs.

temperature for type 1 MTJ samples is much larger than that of the type 2 samples. At $300 \mathrm{~K}$, the $H_{c}$ is about 9 Oe for both types. However, the $H_{c}$ of type 1 rises rapidly to 65 Oe at $13 \mathrm{~K}$, while the $H_{c}$ of type 2 exhibits no significant change.

The center of the MR loops shows a shift from zero fields. This field shift for both types of sample is small. For type 1 MTJ, it is 5 Oe and for type 2 MTJ, it is 1.8 Oe. In both types of samples, a synthetic antiferromagnetic (SAF) structure, $54 \mathrm{FeCo}-9 \mathrm{Ru}-50 \mathrm{CoFeB}$, has been applied to the pinned layer structures to reduce the shift field. ${ }^{19,20}$ However, as the temperature decreases, the coupling field for type 1 MTJs increases to about $8 \mathrm{Oe}$, with no significant change for type 2. The small difference in the Néel coupling (or orange peel coupling) field in these two types of the samples is likely due to the different free layer structures. The additional $50 \AA$ RuTa and $40 \AA \mathrm{CoFeB}$ layers in type 2 MTJs are designed to further control the $\mathrm{CoFeB}$ free layer next to the barrier, so that a linear output can be obtained when patterned into certain shapes and processed accordingly.

\section{B. Voltage dependence of conductance}

$J-V$ curves were measured using a constant voltage source from 14 to $300 \mathrm{~K}$ (Fig. 4). The conductance $G(V)$ curves were obtained by numerically dividing current by voltage. Both parallel and antiparallel configurations were measured at \pm 200 Oe for type 1 and \pm 100 Oe for type 2 . Because the minimum of the $G(V)$ curves has only a small offset from zero bias, we choose the Brinkman-Dynes-Rowell model. ${ }^{21-23}$ This model is given by

$$
\begin{aligned}
G(V) & =\frac{\text { I/area }_{\text {junction }}}{V} \\
& =G_{0}\left[1-\left(\frac{A_{0} \Delta \phi}{16 \bar{\phi}^{3 / 2}}\right)(q V)+\left(\frac{9}{128} \frac{A_{0}^{2}}{\bar{\phi}}\right)(q V)^{2}\right],
\end{aligned}
$$

where $\quad \Delta \phi=\phi_{2}-\phi_{1}, \quad A_{0}=\frac{4 t \sqrt{2 m}}{3 \hbar}, \quad$ and $\quad G_{0}=\left(\frac{q^{2}}{t h^{2}}\right) \sqrt{2 q m \phi}$
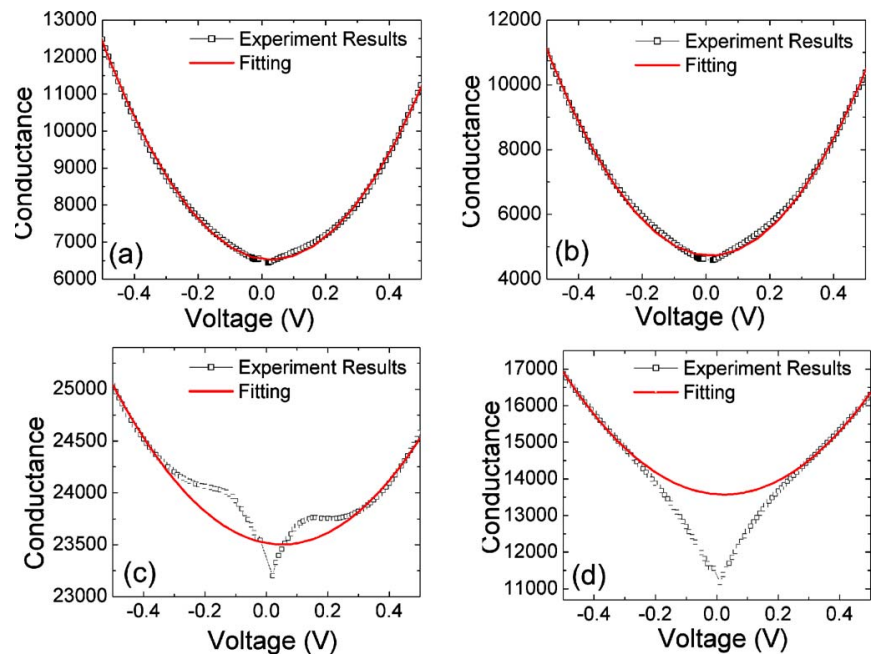

FIG. 4. (Color online) Conductance vs bias voltage at $13 \mathrm{~K}$ for type 1 in the (a) $P$ state and (b) $A P$ state and for type 2 in the (c) $P$ state and (d) $A P$ state.

$\times \exp \left(-\frac{t \overline{8 m q \phi}}{\hbar}\right)$, where $t$ is the barrier thickness in angstroms and $\phi$ is the potential in volts. By fitting the $G(V)$ curves measured at $13 \mathrm{~K}$, we obtained the barrier parameters for two types of samples as shown in Table I. The calculated barrier thickness of type 1 MTJ is larger than that of type 2 MTJ. The barrier height of type 2 MTJ is higher than that of the type 1 MTJ. The calculated barrier thickness of both types of samples is smaller than the starting $\mathrm{Al}$ layer thickness, $12 \AA$. These results indicate that both types of MTJ samples are likely to be either underoxidized or dominated by thinner regions in the barrier that contribute to most of the tunneling. The different values may relate to the different oxygen diffusion processes in these two types of MTJ samples when oxidizing the $\mathrm{Al}$ layer to the $\mathrm{Al}_{2} \mathrm{O}_{3}$ layer. And, from the fitting, the barrier height indicates that the surface roughness in type 1 MTJ may be higher than that of type 2 MTJ samples.

From the fitting curves, it can be seen that the model fits well for type 1 MTJ samples and only fits well in the range of -0.2 to $-0.5 \mathrm{~V}$ and +0.2 to $+0.5 \mathrm{~V}$ for type 2 MTJ samples. As the voltage increases, the conductance of type 2 MTJ samples has a rapid increase in the range of about $200 \mathrm{mV}$, and the increase is more pronounced for the $A P$ alignment. The zero bias anomaly phenomena may be explained by a hot electron. ${ }^{24}$ An electron with excess energy above the Fermi level is called a "hot electron." With an applied bias voltage, those hot electrons will produce collective excitations of local interfaces between the insulating barrier and the ferromagnetic electrodes. The emission of magnons at the interface accounts for the conductance increase as

TABLE I. The fitting barrier parameters of type 1 and type 2 MTJ samples.

\begin{tabular}{lrccc}
\hline \hline & $t_{P}(\AA)$ & $\phi_{P}(\mathrm{eV})$ & $t_{A P}(\AA)$ & $\phi_{A P}(\mathrm{eV})$ \\
\hline Type 1 & 11.3 & 1.28 & 12.7 & 1.03 \\
Type 2 & 6.7 & 3.46 & 7.2 & 3.28 \\
\hline \hline
\end{tabular}



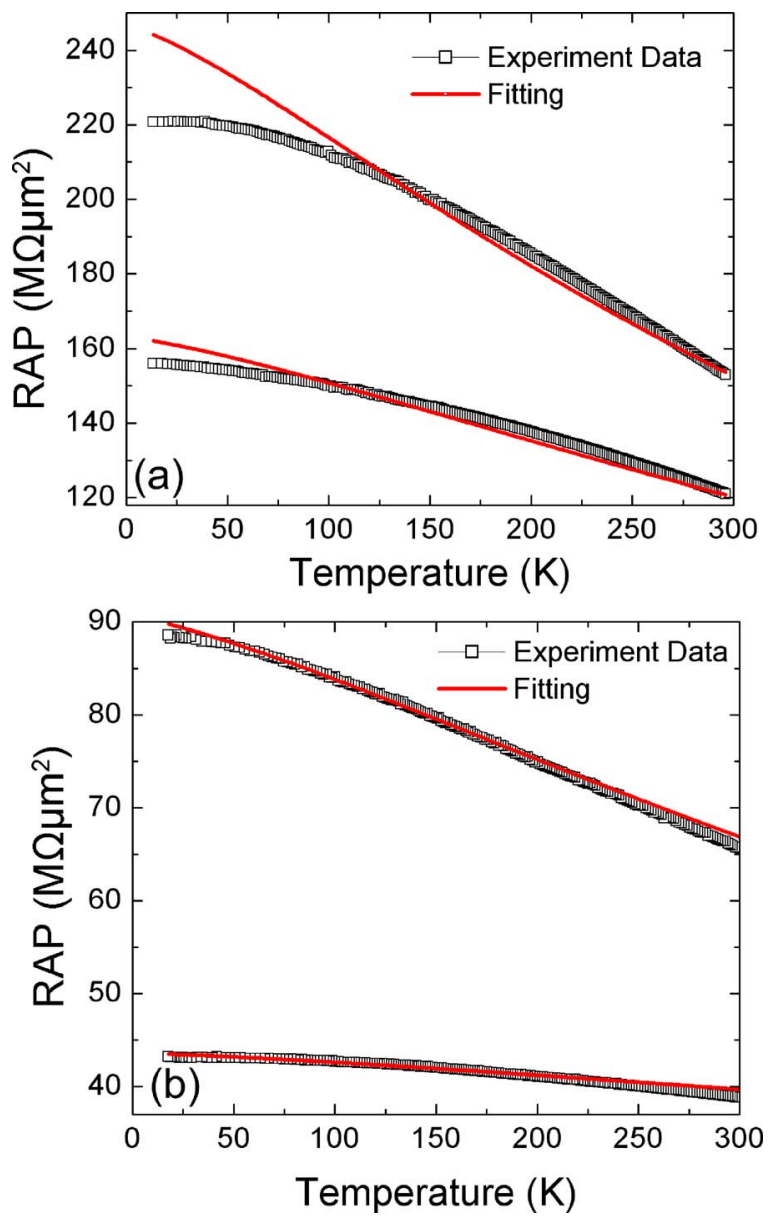

FIG. 5. (Color online) Temperature dependence of the resistance area product (RAP) in the $P$ and $A P$ states for (a) type 1 and (b) type 2 MTJs.

the applied voltage increases. However, we do not have experimental evidence that can clearly illustrate the above models. The observed zero bias anomaly in our type 2 MTJ samples is still not fully understood.

\section{Temperature dependence of resistance}

Figure 5 shows the temperature dependence of RAP results for the two types of samples with a constant bias voltage of $10 \mathrm{mV}$. For type 1 , from 300 to $15 \mathrm{~K}$, the resistance increases $45 \%$ for the antiparallel state and increases $30 \%$ for the parallel state. However, for type 2 it changes $31 \%$ for the antiparallel state, which is much more than the change of $10 \%$ in the parallel state. The dependence of the resistance on temperature is usually explained by elastic and inelastic tunneling. ${ }^{25}$ In this simple model, the temperature dependence of conductance for antiparallel and parallel states is

$$
G_{P}=G_{T}\left[1+P_{1} P_{2}\right]+s T^{1.33},
$$

and

$$
G_{A P}=G_{T}\left[1-P_{1} P_{2}\right]+s T^{1.33},
$$

where $G_{T}=G_{0} \frac{C T}{\sin (C T)}, P_{1}$ and $P_{2}$ are the effective tunneling electron spin polarizations of the two ferromagnetic elec- trodes, and $C=1.387 \times 10^{-4} t / \sqrt{\phi}$, with the barrier thickness $(t)$ in angstroms and the barrier height $(\phi)$ in electron-volts. $G_{0}$ is the conductance of the MTJ at zero temperature. In Eq. (1), the first part represents the elastic tunneling conductance, and the second part represents the inelastic one.

It is assumed that the tunneling spin polarization follows the same temperature dependence as surface magnetization, Bloch $T^{3 / 2}$ law. This means we can write the spin polarization at temperature $T$ as

$$
P(T)=P_{0}\left(1-\alpha T^{3 / 2}\right) .
$$

The parameter $P_{0}$ is the polarization of the electrode at zero temperature. The constant $\alpha$ is a material-dependent constant. It describes the exchange coupling in the direction perpendicular to the surface. The parameter $\alpha$ is generally larger for the surface than the bulk due to surface exchange softening. ${ }^{26}$ The parameter $P_{0}$ is sensitive to disorder and defects in the FM electrodes and FM/I interface. These disorder and defects could be due to interdiffusion at the interface, interface roughness, impurities, and grain boundaries, etc. And, those nonmagnetic or paramagnetic disorders cause the total spin polarization in the free layer to decrease. Therefore, with an increase in disorder, the spin polarization is reduced. From Eq. (1), as polarization increases, $G_{P}$ increases, whereas $G_{A P}$ decreases. Because the free layer and the pinned layer of type 2 MTJ samples are of the same material with similar thicknesses, and sputtered under similar conditions, we assumed they have the same polarization $P_{0}$ and parameter $\alpha$ for fitting our experimental results. For the type 1 MTJ samples, the free layer and the pinned layers are of the same material, but have different thicknesses, so we used different $\alpha$ and $P_{0}$ for the free layer and the pinned layer. In the fitting process for type 1 MTJ samples, we assumed that the polarization $P_{0}$ and parameter $\alpha$ of the pinned layer are the same as that of type 2 MTJ samples, since they are the same material, same film thickness, and same fabrication conditions. In the Shang's model, the TMR increases only with decreasing temperature. Our TMR data for type 1 MTJ samples show a maximum at about $70 \mathrm{~K}$ (Fig. 6), so we fit only the high-temperature data using this model. The values of these parameters from the fitting are listed in Table II.

By computing the TMR ratio by $\left[\left(R_{A P}-R_{P}\right) / R_{P}\right]$, we obtained the temperature dependence of TMR ratio for type 1 and type 2 MTJs from 13 to $300 \mathrm{~K}$. Figure 6 shows that the TMR ratio is around $26.3 \%$ and $69.4 \%$ at room temperature for type $1 \mathrm{MTJ}$ and type $2 \mathrm{MTJ}$, respectively. Before the temperature decreases to about $150 \mathrm{~K}$, the TMR ratio of type 1 MTJ increases and follows the fitting curve well. As the temperature decreases more, the increase becomes much slower and gradually reaches a maximum of $42.7 \%$ at about $70 \mathrm{~K}$ before decreasing to $41.5 \%$ at $13 \mathrm{~K}$. This behavior of the TMR ratio decrease with decreasing temperature at low temperature was also reported by J. H. Lee et al. on their overoxidized MTJs. ${ }^{27}$ The TMR ratio of the type 2 MTJ samples shows a monotonic increase with decreasing temperature. The highest TMR ratio for type 2 MTJ samples is $106.8 \%$ at $13 \mathrm{~K}$. 

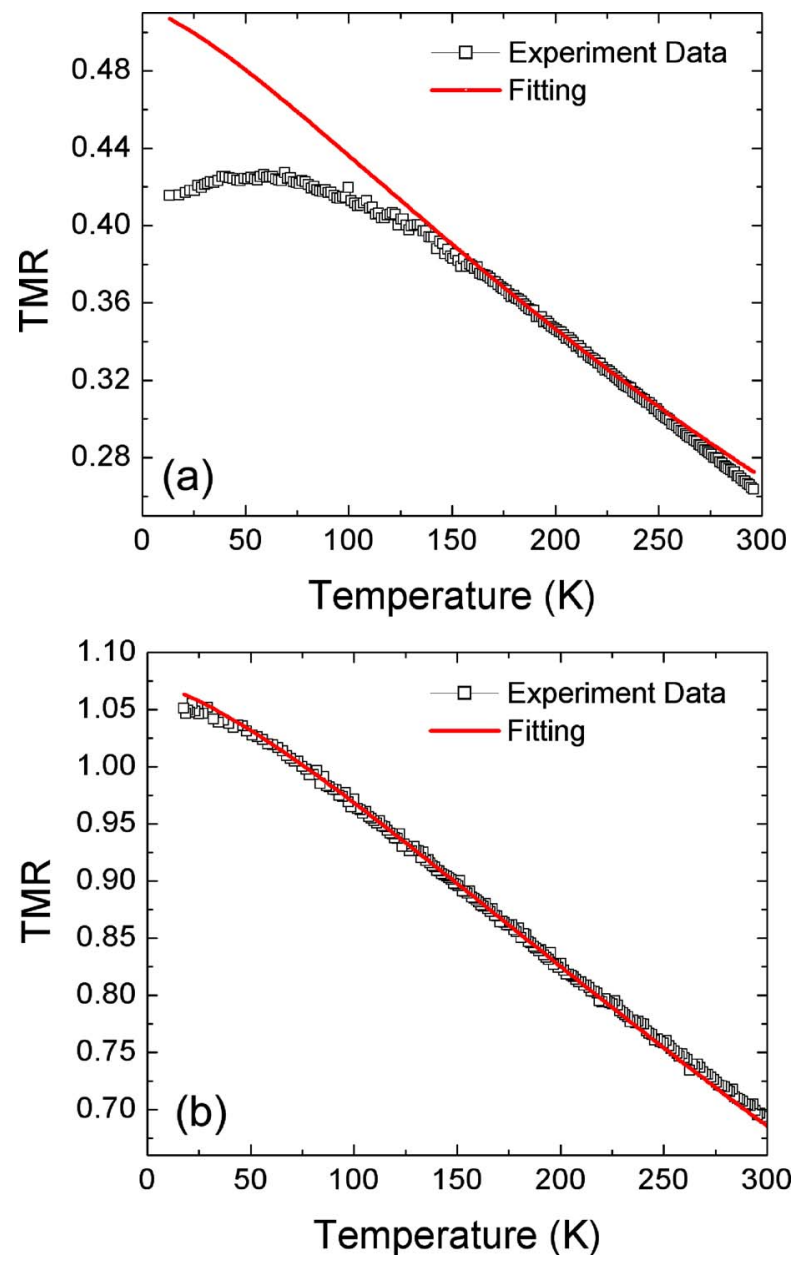

FIG. 6. (Color online) Temperature dependence of TMR for (a) type 1 and (b) type 2 MTJs.

\section{Temperature dependence of bias voltage dependence of magnetoresistance}

Measurement of the voltage dependence of magnetoresistance at different temperatures is shown in Fig. 7. The figure shows a decreasing TMR ratio with increasing bias voltage for both types. This drop may be caused by inelastic scattering by magnon excitations at the ferromagnet/insulator interface which controls the voltage dependence. ${ }^{24} V_{1 / 2}$ (the bias voltage where the TMR ratio reaches half the zero-bias value) is $320 \mathrm{mV}$ at room temperature and $300 \mathrm{mV}$ at $13 \mathrm{~K}$ for type 1. For type 2 it is higher than $500 \mathrm{mV}$ at room temperature and $450 \mathrm{mV}$ at $13 \mathrm{~K}$. $V_{\text {out }}$ (defined as $V_{\text {out }}=V$ $\times$ TMR) is related to the highest signal output from a MTJ device, and it is an important parameter for the device application. The highest output which can be achieved, $V_{\max }$, is

TABLE II. The fitting transport mechanisms and junction quality parameters.

\begin{tabular}{lcccc}
\hline \hline & $G_{0}\left(\Omega \mu \mathrm{m}^{2}\right)^{-1}$ & $P_{0}$ & $\alpha\left(K^{-3 / 2}\right)$ & $s\left(\Omega \mu \mathrm{m}^{2}\right)^{-1} K^{-4 / 3}$ \\
\hline Type 1 & $5.1 \times 10^{-9}$ & $34.5 \%$, & $2.1 \times 10^{-5}$ & $1.1 \times 10^{-12}$ \\
Type 2 & $1.7 \times 10^{-8}$ & $59.1 \%$, & $1.4 \times 10^{-5}$ & $1.5 \times 10^{-12}$ \\
\hline \hline
\end{tabular}

$50 \mathrm{mV}$ at $300 \mathrm{~K}$ and $70 \mathrm{mV}$ at $13 \mathrm{~K}$ for type 1 , while for type $2, V_{\max }$ is higher than $205 \mathrm{mV}$ at $300 \mathrm{~K}$ and higher than $256 \mathrm{mV}$ at $18 \mathrm{~K}$. From Table I, we have shown that the barrier height of type 2 samples is much higher than that of type 1 , while the barrier thickness of type 2 is much thinner than that of type 1 . These results indicate that the samples with a higher barrier height have a higher $V_{1 / 2}$, while $V_{1 / 2}$ may not relate to the barrier thickness.

\section{DISCUSSION}

From the MTJ layer structures, the main mechanisms that are responsible for the TMR behaviors should be related to the ferromagnetic layers, the barrier layer, and the two FM/I interfaces. We observed very different TMR ratios in the type 1 and type 2 MTJ samples. The TMR ratio is about $26 \%$ and $69 \%$ at room temperature for type 1 and type $2 \mathrm{MTJ}$, respectively. Since our two types of sample are different only in the free layer structures, the factors contributing to the magnetoresistance behavior changes should come mostly from the detailed magnetization orientation of free layer structures and/or the interface between the free layer and the insulating layer. From the analysis of our voltage and temperature dependence of resistance and magnetoresistance in these MTJs, we discuss the effects of magnetic behavior of the free layers, the interface between the free layer and the insulating layer, and barrier qualities.

The free layers of both types are made of the same material, but have different thicknesses. The thickness of the free layer is as thin as $8 \AA$ for type 1 , which corresponds to only a few monolayers. From previous studies, the films with thicknesses in this range may be superparamagnetic, nonmagnetic, or ferromagnetic. ${ }^{28-31}$ The thin free layer of our type 1 MTJ samples is mostly ferromagnetic, which shows hysteresis. Since the free layer of type 1 is 5 times thinner than that of type 2, we would then expect there to be more defects in the free layer of type 1 than in that of type 2 . The defects could cause incoherent nucleation, domain-wall pinning in the free layer and/or the interface between the free layer and the insulating layer. Evidence of the existence of more magnetic inhomogeneous regions in the free magnetic layer and/or the interface between the free layer and the insulating layer of type 1 MTJ samples than in that of type 2 can be illustrated by the temperature dependence of $H_{c}$, the squareness of the loop, the Néel coupling field, the temperature dependence of resistance in the $P$ and $A P$ states, the polarization of the electrode at the zero temperature $P_{0}$, and the spin exchange stiffness constant $\alpha$.

From Fig. 2, we observe the larger temperature variation of $H_{c}$ and the lower $S$ value in type 1 MTJ samples than that of type 2. This indicates that the magnetization of the free layer and/or the interface between the free layer and the insulating layer does not switch with the field as homogeneously as type 2 does. The Néel coupling field at $300 \mathrm{~K}$ for type 1 and type 2 MTJ is 5 and $1.8 \mathrm{Oe}$, respectively. The small difference in Néel coupling field in these two types of samples is likely due to the different free layer structures, since the amplitude of the coupling field is related to the increase in magnetic roughness. The increase in the Néel 

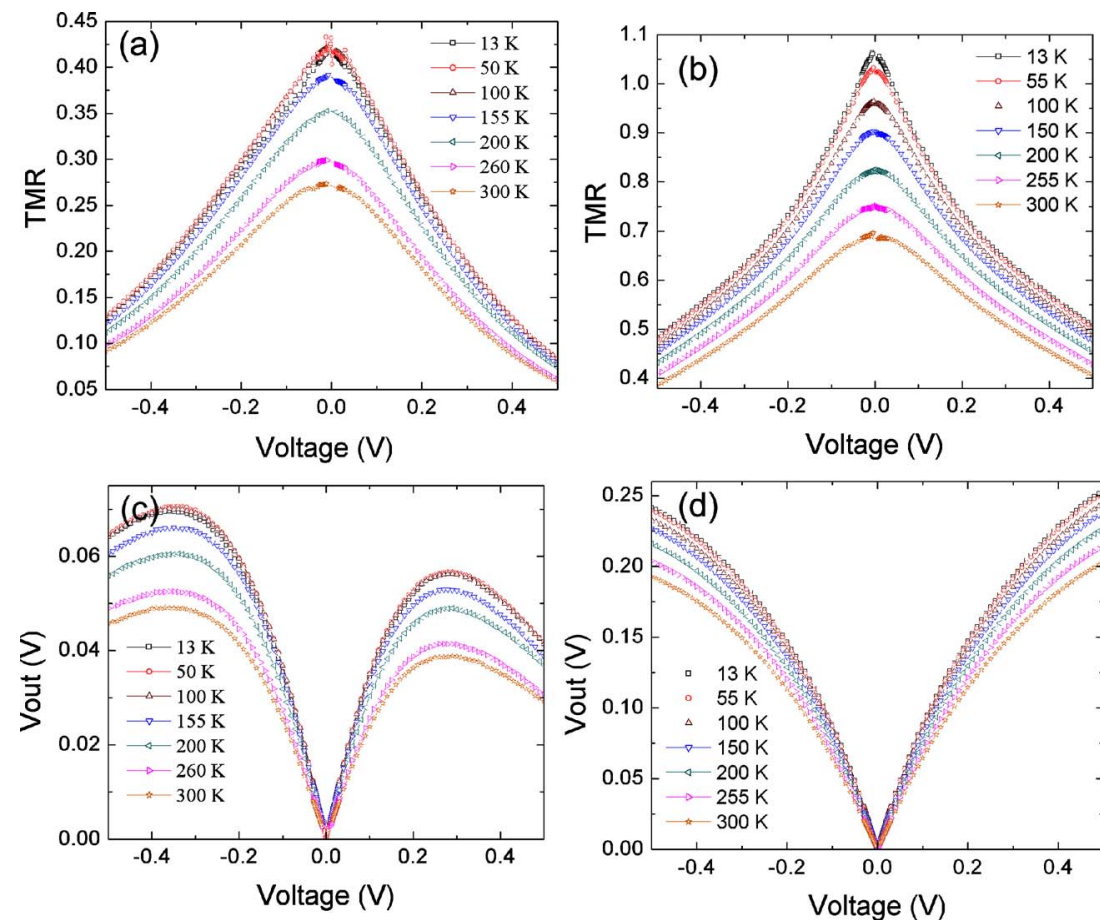

FIG. 7. (Color online) Voltage dependence of TMR at different temperatures for (a) type 1 and (b) type 2 samples and voltage dependence of $\mathrm{V}_{\text {out }}$ (defined as $V^{*} \mathrm{TMR}$ ) for (c) type 1 and (d) type 2 samples. The direction of bias voltage is defined with respect to upper electrode. coupling field in type 1 MTJ samples indicates the possibility that there is more magnetic roughness in type 1 MTJ samples than in that of type 2 .

As shown in Fig. 5, the temperature dependence of resistance for type 1 MTJ samples in the $A P$ state and in the $P$ state increases $45 \%$ and $30 \%$, respectively. The temperature dependence of resistance for type 2 MTJ samples in the AP state and in the $P$ state increases $31 \%$ and $10 \%$, respectively. The dependence of the resistance on temperature is usually explained by elastic and inelastic tunneling. ${ }^{25}$ The increasing TMR ratio with decreasing temperature is caused by the fact that the increase in $R_{A P}$ occurs more quickly than in $R_{P}$. This is because $R_{A P}$ decreases while $R_{P}$ increases with increasing temperature when there are magnetic disorders in the MTJ samples. Thermal assist tunneling will decrease both $R_{A P}$ and $R_{P}$ with increasing temperature. ${ }^{15}$ Due to a combination of these two effects, the increase of resistance in the $A P$ state with decreasing temperature is more than in the $P$ state. The rate of decreasing TMR ratio is larger for type 1 MTJ samples than for type 2 . The rate of decrease is calculated as follows. From Fig. 6, the TMR ratio is $26.3 \%$ at room temperature and $42.7 \%$ at about $70 \mathrm{~K}$ for type $1 \mathrm{MTJ}$ samples (i.e., the TMR rate of change from 70 to $300 \mathrm{~K}$ is $38 \%$ ). The TMR ratio is $69.4 \%$ at room temperature and $98.7 \%$ at about $70 \mathrm{~K}$ for type $2 \mathrm{MTJ}$ samples (i.e., the TMR ratio rate of change from 70 to $300 \mathrm{~K}$ is $30 \%$ ). The difference in the rate change of the TMR ratio implies that there are more spin disorders in the type 1 MTJ samples than in type 2.

The parameter $P_{0}$ is the spin polarization of the electrode at zero temperature. The fitting $P_{0}$ of type 1 MTJ samples is less than that of type 2 MTJ samples. The parameter $P_{0}$ is sensitive to disorder and defects in the FM electrodes and $\mathrm{FM} / I$ interface. A possible source of disorder and defects in the free layer is the interdiffusion between the bottom $\mathrm{Ru}$ and the $\mathrm{CoFeB}$ layer. The nonmagnetic $\mathrm{Ru}$ may cause a decrease in the spin polarization in the free layer. From Table II, the $P_{0}$ of the type 2 MTJ samples is about $59.1 \%$, which is almost the maximum limit for the $3 d$ magnetic material. This implies the spins in the free layer of type 2 MTJ samples are strongly coupled and well-aligned. Since the free layer thicknesses in the type 1 and type 2 MTJ samples are different, the type 2 MTJ samples may not be affected by this small interdiffusion at the bottom of the free layer. The constant $\alpha$ is a material-dependent constant which relates to the softening of the spin exchange coupling. The parameter $\alpha$ is generally larger for the surface than the bulk due to surface exchange softening. ${ }^{26}$ From Table II, the magnitude of $\alpha$ is $2.1 \times 10^{-5} \mathrm{~K}^{-3 / 2}$ and $1.4 \times 10^{-5} \mathrm{~K}^{-3 / 2}$ for type 1 and type 2 MTJ samples, respectively. The values have the same order of magnitude with most amorphous alloys. The fitting parameter $\alpha$ of type 1 MTJ samples is larger than that of type 2 . This clearly indicates that the type 1 MTJ sample has more magnetic inhomogeneous regions than the type 2 MTJ sample. Those magnetic inhomogeneous distributions of local spins may be caused by disorder or discontinuity as well as roughness in the thin free layer of type 1 MTJs. In addition, it may also be $\mathrm{CoFeB}$ isolated at the interface between the thin free layer of the type 1 sample and the Ru layer due to interdifussion and/or $\mathrm{Al}_{2} \mathrm{O}_{3}$ layer due to partially oxidization.

From Fig. 3, we observe that the squareness $(S)$ values of type 1 MTJ samples decrease rapidly at about $70 \mathrm{~K}$ while the $S$ values of type 2 samples keep about the same. The reduction in $S$ value indicates that more additional magnetic pinning sites may exist in the type 1 MTJ at low temperature. This pinning may be due to additional magnetic components that occur at low temperatures or due to the temperature dependence of the magnetic anisotropy. This makes the whole $\mathrm{CoFeB}$ film difficult to align with the external field, especially at low temperatures. This may be explained by an 
existing spin-glass-like phase, which is hardly aligned under spin freeze temperature. The incoherent rotations at low temperature increase the magnetic roughness in the free layer, which causes the $H_{c}$ to increase and the Néel exchange coupling field of type 1 MTJ to increase with decreasing temperature.

Figure 6 shows that there is a maximum at about $70 \mathrm{~K}$ in the TMR ratio versus temperature curve for type 1 MTJ samples. The possible reason for the maximum may be due to spin-glass regions or inhomogeneous distribution of local spin in the free layer that has spin reorientation with the changing temperature. When the temperature is higher than the spin freeze temperature, those regions act paramagnetically and the magnetization will decrease with a temperature increase. If the sample is cooled below the spin freeze temperature, the magnetization will be frozen randomly and the average magnetization will be reduced with decreasing temperature. At low temperature, the total effect of both the spinglass-like regions and the ferromagnetic regions may result in increasing the spin polarization of the whole free layer, $P_{1}$, with increasing temperature.

From Eq. (1), we can express the TMR as TMR $=\frac{1}{\left(1+s T^{1.33}\right) / P_{1} P_{2}-1}$. If the increase in $P_{1}$ is large enough to make the increase in the product $P_{1} P_{2}$ larger than the increase in $1+\mathrm{sT}^{1.33}$, then the TMR will increase with a temperature increase. When the temperature is higher than the spin freeze temperature, both magnetic disorder and the spin glass cause the decrease in the spin polarization. This causes the resistance decrease in the $A P$ state to be more than in the $P$ state with a temperature increase, resulting in a TMR decrease.

The barrier quality can be revealed by the study of the temperature dependence of resistance of both types of samples. The fitting parameter $s$ [in Eq. (1)] describes the temperature dependence of the spin-independent conductance. Some mechanisms may cause the spin-independent conductance. Among them, the most important two are hopping conductance due to imperfections in the barrier and pinholes in the barrier layer. The two types of samples have similar small value of $s,{ }^{23,32}$ which indicates both types of samples have a clean interface between the FM/I interface.

\section{CONCLUSION}

We studied the voltage and temperature dependence of resistance and magnetoresistance of two types of MTJs. These two types of MTJ samples have different free layer structures but the same pinned structures and the same material for free and reference layers. We observed quite different TMR ratios for these two types of samples. For type 2, a TMR ratio as high as $107 \%$ was detected. We discuss the effects of magnetic behavior of the free layers, barrier qualities, and barrier interfaces. From the analysis of our results, we conclude that: (1) There are more magnetic inhomogeneous regions in the free magnetic layer and/or at the interface between the free layer and the insulating layer of type 1 MTJ samples than in that of type 2 MTJ samples; (2)There are possible additional spin-glass-like states that occurs at the interface between the free layer and the insulating layer in the type 1 MTJ sample at low temperature; (3) Type 2 MTJ samples have thinner barrier thickness, higher barrier potentials, and higher output $V_{\max }$ than the type 1 MTJ samples. These results clearly indicate that the micromagnetization orientation in the free layer and the FM/I interface play an important role in determining the TMR ratio in these two types of MTJ samples.

\section{ACKNOWLEDGMENTS}

We thank E. Y. Tsymbal for useful discussion. This research was supported by the NSF MRSEC Award DMR0213808, DMI-9961165, DMI-03-19974, and MDA under DTRA01-99-C-0110. The project is also supported by ARO DAAD Grant No. 19-03-1-0298, the W. M. Keck Foundation, and the Nebraska Research Initiative at the University of Nebraska.
${ }^{1}$ M. Julliere, Phys. Lett. 54A, 225 (1975);

${ }^{2}$ J. S. Moodera, L. R. Kinder, T. M. Wong, and R. Meservey, Phys. Rev. Lett. 74, 3273 (1995)

${ }^{3}$ T. Miyazaki and N. J. Tezuka, J. Magn. Magn. Mater. 139, L231 (1995).

${ }^{4}$ P. Tedrow and R. Meservey, Phys. Rev. B 7, 318 (1973).

${ }^{5}$ Warren E. Pickett, and Jagadeesh S. Moodera, Phys. Today 54, 39 (2001).

${ }^{6}$ J.-H. Park, E. Vescovo, H.-J. Kim, C. Kwon, R. Ramesh, and T. Venkatesan, Nature (London) 392, 794 (1998).

${ }^{7}$ D. Ristoiu, J. P. Nozières, C. N. Borca, T. Komesu, H.-K. Jeong, and P. A. Dowben, Europhys. Lett. 49, 624 (2000).

${ }^{8}$ Y. Ji, G. J. Strijkers, F. Y. Yang, C. L. Chien, J. M. Byers, A. Anguelouch, G. Xiao, and A. Gupta, Phys. Rev. Lett. 86, 5585 (2001).

${ }^{9}$ J. S. Parker, S. M. Watts, P. G. Ivanov, and P. Xiong, Phys. Rev. Lett. 88, 196601 (2002).
${ }^{10}$ Y. Lu, X. W. Li, G. Q. Gong, Gang Xiao, A. Gupta, P. Lecoeur, J. Z. Sun, Y. Y. Wang, and V. P. Dravid, Phys. Rev. B 54, R8357 (1996).

${ }^{11}$ M. Viret, M. Drouet, J. Nassar, J. P. Contour, C. Fermon, and A. Fert, Europhys. Lett. 39, 545 (1997).

${ }^{12}$ R. Jansen and J. S. Moodera, Phys. Rev. B 61, 9047 (2000).

${ }^{13}$ E. Yu. Tsymbal and D. G. Pettifor, J. Appl. Phys. 85, 5801 (1999).

${ }^{14}$ Dexin Wang, Cathy Nordman, James M. Daughton, Zhenghong Qian, and Jonathon Fink, IEEE Trans. Magn. 40, 2269 (2004).

${ }^{15}$ Stuart S. P. Parkin, Christian Kaiser, Alex Panchula, Philip M. Rice, Brian Hughes, Mahesh Smant, and See-Hun Yang, Nature (London) 3, 862 (2004).

${ }^{16}$ Shinji Yuasa, Taro Nagahama, Akio Fukushima, Yoshishige Suzuki, and Koji Ando, Nature (London) 3, 868 (2004).

${ }^{17}$ Dexin Wang, Zhenghong Qian, James M. Daughton, Cathy Nordman, Mark Tondra, Daniel Reed, and David Brownell, J. Appl. 
Phys. 89, 6754 (2001).

${ }^{18}$ D. Wang, M. Tondra, J. M. Daughton, C. Nordman, and A. V. Pohm, J. Appl. Phys. 85, 5255 (1999).

${ }^{19}$ Dexin Wang, James M. Daughton, Zhenghong Qian, Cathy Nordman, Mark Tondra, and Art Phom, J. Appl. Phys. 93, 8558 (2003).

${ }^{20}$ B. D. Schrag, A. Anguelouch, S. Ingvarsson, Gang Xiao, Yu Lu, P. L. Trouilloud, A. Gupta, R. A. Wanner, W. J. Gallagher, P. M. Rice, and S. S. P. Parkin, Appl. Phys. Lett. 77, 2373 (2000).

${ }^{21}$ John G. Simmons, J. Appl. Phys. 35, 2655 (1964).

${ }^{22}$ W. F. Brinkman, R. C. Dynes, and J. M. Rowell, J. Appl. Phys. 41, 1915 (1970).

${ }^{23}$ Bryan Oliver and Janusz Nowak, J. Appl. Phys. 95, 546 (2004).

${ }^{24}$ S. Zhang, P. M. Levy, A. C. Marley, and S. S. P. Parkin, Phys. Rev. Lett. 79, 3744 (1997).

${ }^{25}$ Chang He Shang, Janusz Nowak, Ronnie Jansen, and Jagadeesh S. Moodera, Phys. Rev. B 58, R2917 (1998).

${ }^{26}$ D. Mauri, D. Scholl, H. C. Siegmann, and E. Kay, Phys. Rev.
Lett. 61, 758 (1988).

${ }^{27}$ J. H. Lee, K. I. Lee., W. L. Lee, K. H. Shin, J. S. Lee, K. Phie, and B. C. Lee, J. Appl. Phys. 91, 7956 (2002); K. I. Lee, J. H. Lee, W. L. Lee, K. H. Shin, Y. B. Sung, J. G. Ha, K. Rhie, and B. C. Lee, J. Appl. Phys. 91, 7959 (2002).

${ }^{28}$ D. Wang, J. M. Daughton, K. Bussmann, and G. A. Prinz, J. Appl. Phys. 83, 7034 (1998).

${ }^{29}$ R. Schad, H. Alouach, J. W. Harrell, M. Shamsuzzoha, and D. Wang, J. Appl. Phys. 93, 8561 (2003).

${ }^{30}$ C.-Y. Hung, M. Mao, S. Funada, T. Schneider, L. Miloslavsky, M. Miller, C. Qian, and H. C. Tong, J. Appl. Phys. 87, 6618 (2000).

${ }^{31}$ Y. Luo, M. Esseling, A. Käufler, K. Samwer, T. Dimopoulos, G. Gieres, M. Vieth, M. Rührig, J. Wecker, C. Rudolf, T. Niermann, and M. Seibt, Phys. Rev. B 72, 014426 (2005).

${ }^{32}$ T. Hagler, R. Kinder, and G. Bayreuther, J. Appl. Phys. 89, 7570 (2001). 